\title{
Characterisation of virulence genes in methicillin susceptible and resistant Staphylococcus aureus isolates from a paediatric population in a university hospital of Medellín, Colombia
}

\author{
Judy Natalia Jiménez ${ }^{1 /+}$, Ana María Ocampo', Johanna Marcela Vanegas',Erika Andrea Rodríguez, \\ Carlos Guillermo Garcés ${ }^{2,3}$, Luz Adriana Patiño², Sigifredo Ospina², Margarita María Correa
}

${ }^{1}$ Grupo de Microbiología Molecular, Escuela de Microbiología ${ }^{3}$ Grupo de Investigación Clínica en Enfermedades del Niño y del Adolescente, Universidad de Antioquia, Calle 67 53-108, 1226 Medellín, Colombia ${ }^{2}$ Hospital Universitario, San Vicente Fundación, Medellín, Colombia

Virulence and antibiotic resistance are significant determinants of the types of infections caused by Staphylococcus aureus and paediatric groups remain among the most commonly affected populations. The goal of this study was to characterise virulence genes of methicillin-susceptible S. aureus (MSSA) and methicillin-resistant S. aureus (MRSA) strains isolated from a paediatric population of a Colombian University Hospital during 2009. Sixty MSSA and MRSA isolates were obtained from paediatric patients between zero-14 years. We identified the genes encoding virulence factors, which included Panton-Valentine leucocidine (PVL), staphylococcal enterotoxins A-E, exfoliative toxins A and B and toxic shock syndrome toxin 1. Typing of the staphylococcal chromosome cassette mec (SCCmec) was performed in MRSA strains. The virulence genes were more diverse and frequent in MSSA than in MRSA isolates $(83 \%$ vs. $73 \%)$. MRSA strains harboured SCCmec types IVc (60\%), I (30\%), IVa (7\%) and V (3\%). SCCmec type IVc isolates frequently carried the PVL encoding genes and harboured virulence determinants resembling susceptible strains while SCCmec type I isolates were often negative. PVL was not exclusive to skin and soft tissue infections. As previously suggested, these differences in the distribution of virulence factor genes may be due to the fitness cost associated with methicillin resistance.

Key words: Staphylococcus aureus - MRSA - MSSA - virulence factors

The success of Staphylococcus aureus as a pathogen is in part due to its ability to express a variety of virulence factors, both structural and secreted, that mediate host colonisation, tissue invasion and dissemination (Gordon \& Lowy 2008). In addition, S. aureus has an exceptional ability to develop resistance to antimicrobial agents (Chambers \& Deleo 2009). Among the most known secreted virulence factors is a large family of superantigen exotoxins that include the staphylococcal enterotoxins, which cause food poisoning, toxic shock syndrome toxin 1 (tst -1$)$ and the exfoliative toxins (eta and etb), which are implicated in staphylococcal scalded-skin syndrome (John \& Schreiber 2006, Tristan et al. 2007). An important virulence factor produced by many methicillin-resistant S. aureus (MRSA) strains is the Panton-Valentine leucocidine (PVL), a bicomponent pore-forming cytolytic toxin that targets cell membranes of leukocytes (McClure et al. 2006), which is associated with skin and soft tissue infections (SSTI), necrotising pneumonia and epidemiologically linked to community-associated MRSA (CAMRSA) (Chambers 2005, DeLeo et al. 2009).

S. aureus and in particular CA-MRSA, has become a successful pathogen within the paediatric population. A

Financial support: CODI (8700-039), COLCIENCIAS (1115-459-21442) JNJ received financial support for doctoral training.

+ Corresponding author: judynatalia@yahoo.com

Received 22 March 2011

Accepted 31 August 2011 dramatic increase in community-MRSA infections has been reported in healthy children (Herold et al. 1998, Bocchini et al. 2006, Stankovic \& Mahajan 2006, Faden et al. 2007, Baker 2010, Wu et al. 2010).

This could be explained by the high rates of anterior nares' carriage in the paediatric population, which is associated with subsequent infection (Miller \& Diep 2008, Lee et al. 2009).

There is significant controversy on the virulence of methicillin-susceptible $S$. aureus (MSSA) vs. MRSA isolates; both have enormous capacity for virulence and pathogenicity that enables them to reach high rates of infection (Gould 2006). However, they differ in their genotypes, geographical distribution and the infections they cause (Kim et al. 2006).

In Colombia, $S$. aureus represents an important and increasing health problem, yet little is known about the virulence factors present in MSSA and MRSA isolates. Therefore, the aim of this study was to determine the frequencies and profiles of virulence factors in a MSSA and MRSA strain collection obtained from the paediatric population of a Colombian University Hospital in 2009. This information is important as it could facilitate the establishment of more effective infection control measures in this institution.

\section{SUBJECTS, MATERIALS AND METHODS}

Study population - An observational cross-sectional study was conducted during 2009 at the Hospital Universitario San Vicente Fundación, a fourth-level care centre located in Medellín, the second largest city of 
Colombia. The sample size was calculated based on the records of paediatric MRSA isolates during 2008, which numbered 30 isolates. Therefore, a sample size of $60 \mathrm{~S}$. aureus isolates, 30 MRSA and 30 MSSA, was taken to establish comparison values. The isolates included in the study were randomly selected from children aged zero14 years in the Paediatric Department of the institution. Clinical and epidemiological information was obtained from the medical records and included data such as gender, age and type of infection. Informed consent to participate in the study was obtained from each patient's parent or guardian.

Bacterial strain identification and molecular confirmation of S. aureus and methicillin resistance - Identification of $S$. aureus was conducted by standard laboratory methods based on colony morphology in sheep blood agar and positive catalase and coagulase tests. Methicillin resistance was assessed in accordance with Clinical and Laboratory Standards Institute guidelines (CLSI 2010).

For molecular confirmation of S. aureus and methicillin resistance, the DNA was extracted from the isolates using the Wizard Genomic DNA purification kit (Promega) according to the manufacturer's instructions with $10 \mathrm{mg} /$ $\mathrm{mL}$ of Chicken Egg White Lysozyme (Sigma Aldrich). The presence of the mecA and fem $A$ genes was verified by polymerase chain reaction (PCR) according to a protocol previously described (Mehrotra et al. 2000).

Staphylococcal chromosome cassette mec (SCCmec) typing and detection of toxin genes - SCCmec types and subtypes were determined by using a set of six multiplex PCR reactions according to a previously reported protocol (Kondo et al. 2007). The genes encoding staphylococcal enterotoxins (sea, seb, sec, sed, see), tst-1, eta and etb were detected by multiplex PCR (Mehrotra et al. 2000). Amplification of the genes for the PVL (lukS/F-PV) was performed as previously reported (McClure et al. 2006).

Statistical analysis - Categorical variables were compared using the chi-square test or Fisher's exact test and $p$ values of $\leq 0.05$ were considered statistically significant. Statistical analysis was carried out using the SPSS ${ }^{\circledR}$ v15.0 software package.

Ethics - The study was approved by a bioethics committee at Universidad de Antioquia, Medellín (approval 08036172).

\section{RESULTS}

Description of MRSA infections and epidemiologic characteristics - Among the 60 patients included in the study, $44(73.3 \%)$ were males and $39(65 \%)$ were $\geq 1$ year old, with an average of $5.4 \pm 4.3$ years. Patients were distributed into the following services: paediatric ward (37; $62 \%)$, paediatric intensive care unit $(8 ; 13 \%)$, orthopaedic ward $(6 ; 10 \%)$, emergency ward $(4 ; 6.7 \%)$, surgery (4; $6.7 \%)$ and other services $(1,2 \%)$. Isolates were most commonly obtained from SSTI (27; 45\%), followed by pneumonia $(8 ; 13.3 \%)$, surgical site infection (SSI) $(6$; $10 \%)$, central venous catheter-related bacteraemia (5; $8.3 \%)$, primary bacteraemia $(3 ; 5 \%)$, arthritis $(3 ; 5 \%)$, osteomyelitis $(1 ; 1.7 \%)$ and other infections $(7 ; 11.7 \%)$, including urinary tract infections, conjunctivitis, meningitis, otitis, tracheitis, orbital cellulitis and infected pulmonary sequestration.

MSSA isolates and virulence gene profiles - Most MSSA isolates (83.3\%) carried one or more virulence genes simultaneously. lukS/F-PV genes were detected in $11(37 \%)$ of these isolates and staphylococcal enterotoxin genes sea, seb, sec, sed and see were commonly observed. seb was the most frequently detected enterotoxin, which was present in $40 \%$ of the MSSA isolates, followed by sed (33\%), while, eta and etb were not detected (Fig. 1, Table).

MRSA SCCmec types and virulence gene profiles Of the 30 MRSA isolates, $60 \%$ carried SCCmec type IVc (18), 30\% type I (9), 7\% type IVa (2) and 3\% type V (1). Other SCCmec types were not detected. Twenty two $(73 \%)$ MRSA isolates carried one or more virulence genes simultaneously. The SCCmec types showed diverse virulence gene patterns (Table). Those carrying SCCmec IV had one or more virulence genes, similar to MSSA isolates.

lukS/F-PV genes were present in all SCCmec types, but they were more frequently observed in SCCmec IVc isolates (94\%). The staphylococcal enterotoxin genes seb, sed and see were less common and appeared only in SCCmec IV strains, while sea and sec were not detected in any of the MRSA isolates. eta was present at a very low frequency: it was only detected in a SCCmec V strain, while $e t b$ was not detected at all.

Only two (22\%) of the nine SCCmec type I isolates were positive for $l u k S / F-P V$ and all nine were negative for any other toxin gene (Fig. 1, Table).

The bivariate analysis showed statistically significant differences $(p<0.05)$ in the presence of the following virulence genes between MSSA-MRSA isolates: lukS/F-PV (37\% vs. $73 \%$; $\mathrm{p}=0.004)$, seb ( $40 \%$ vs. $7 \%$; $\mathrm{p}=0.002)$ and $\operatorname{sed}(33 \%$ vs. $7 \% ; \mathrm{p}=0.010)($ Table).

Distribution of virulence genes and type of infection - The distribution of virulence genes varied with the type of infection and the strain causing it. In all types

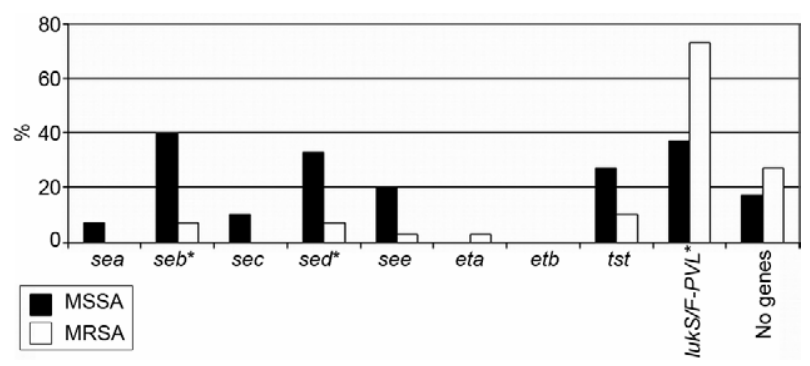

Fig. 1: percentage distribution of virulence genes encoding staphylococcal enterotoxins genes A-E (sea, seb, sec, sed and see), exfoliative toxins A and B (eta and etb), toxic shock syndrome toxin 1 (tst) and Panton-Valentine leucocidine (lukS/F-PV) in methicillin-susceptible Staphylococcus aureus (MSSA) and methicillin-resistant S. aureus (MRSA). Asterisk means $\mathrm{p} \leq 0.05$, by chi-square or Fisher's exact test. 
TABLE

Absolute and relative distribution of staphylococcal virulence genes, enterotoxins (sea, seb, sec, sed, see) the exfoliative toxins (eta and etb), toxic shock syndrome toxin 1 (tst) and Panton-Valentine leukocidin (lukS/F-PV) according to methicillin-resistant Staphylococcus aureus (MRSA) and methicillin-susceptible S. aureus (MSSA) isolates and staphylococcal chromosome cassette mec ( $\mathrm{SCCmec}$ ) types

\begin{tabular}{|c|c|c|c|c|c|c|c|c|}
\hline \multirow[b]{2}{*}{ Gene } & \multirow[b]{2}{*}{$\begin{array}{c}\text { Total } \\
(\mathrm{n}=60) \\
\mathrm{n}(\%)\end{array}$} & \multirow[b]{2}{*}{$\begin{array}{c}\text { MSSA } \\
(\mathrm{n}=30) \\
\mathrm{n}(\%)\end{array}$} & \multirow[b]{2}{*}{$\begin{array}{c}\text { MRSA } \\
(\mathrm{n}=30) \\
\mathrm{n}(\%)\end{array}$} & \multirow[b]{2}{*}{$\mathrm{p}^{a}$} & \multicolumn{4}{|c|}{ MRSA SCCmec types } \\
\hline & & & & & $\begin{array}{c}\mathrm{I} \\
(\mathrm{n}=9) \\
\mathrm{n}(\%)\end{array}$ & $\begin{array}{c}\text { IVa } \\
(\mathrm{n}=2) \\
\mathrm{n}(\%)\end{array}$ & $\begin{array}{c}\text { IVc } \\
(\mathrm{n}=18) \\
\mathrm{n}(\%)\end{array}$ & $\begin{array}{c}\mathrm{V} \\
(\mathrm{n}=1) \\
\mathrm{n}(\%)\end{array}$ \\
\hline One or more & 47 (78) & $25(83)$ & $22(73)$ & NS & $2(22)$ & $2(100)$ & $17(94)$ & $1(100)$ \\
\hline sea & $2(3)$ & $2(7)$ & $0(0)$ & NS & $0(0)$ & $0(0)$ & $0(0)$ & $0(0)$ \\
\hline$s e b$ & $14(23)$ & $12(40)$ & $2(7)$ & 0.002 & $0(0)$ & $0(0)$ & $2(11)$ & $0(0)$ \\
\hline $\sec$ & $3(5)$ & $3(10)$ & $0(0)$ & NS & $0(0)$ & $0(0)$ & $0(0)$ & $0(0)$ \\
\hline sed & $12(20)$ & $10(33)$ & $2(7)$ & 0.010 & $0(0)$ & $2(100)$ & $0(0)$ & $0(0)$ \\
\hline see & $7(12)$ & $6(20)$ & $1(3)$ & NS & $0(0)$ & $1(50)$ & $0(0)$ & $0(0)$ \\
\hline eta & $1(2)$ & $0(0)$ & $1(3)$ & NS & $0(0)$ & $0(0)$ & $0(0)$ & $1(100)$ \\
\hline$e t b$ & $0(0)$ & $0(0)$ & $0(0)$ & - & $0(0)$ & $0(0)$ & $0(0)$ & $0(0)$ \\
\hline tst & $11(18)$ & $8(27)$ & $3(10)$ & NS & 0 & $1(50)$ & $2(11)$ & $0(0)$ \\
\hline$l u k S / F-P V$ & $33(55)$ & $11(37)$ & $22(73)$ & 0.004 & $2(22)$ & $2(100)$ & 17 (94) & $1(100)$ \\
\hline No genes & $13(22)$ & $5(17)$ & $8(27)$ & NS & $7(78)$ & $0(0)$ & 1(6) & $0(0)$ \\
\hline
\end{tabular}

$a$ : by chi-square or Fisher's exact test. NS: not significant.

of MRSA infections, the lukS/F-PV genes were present. Pneumonia infections were predominantly caused by MRSA strains, with PVL genes detected in $67 \%$ of them. Skin and soft tissue and SSI were mainly due to MSSA strains that harboured most of the virulence genes assessed, except for the exfoliative toxin genes. MSSA strains causing blood stream infections (BSI) and arthritis simultaneously carried several virulence genes. MSSA from BSI harboured sed (100\%), tst (100\%) and lukS/F-PV (100\%) and arthritis-associated MSSA presented sed $(100 \%)$ and see $(100 \%)$. The resistant strains causing the same type of infections carried only PVL genes present in $50 \%$ and $100 \%$ of the isolates, respectively. eta was only detected in $50 \%$ of MRSA isolates that caused catheter-related BSI. A single case of osteomyelitis was detected, caused by a SCCmec IVc isolate carrying only PVL genes (Figs 2, 3).

\section{DISCUSSION}

The clinical outcome of $S$. aureus infections is influenced by both the presence of antimicrobial resistance and virulence factors. It has been suggested that the acquisition of antibiotic resistance in $S$. aureus involves changes in virulence factor secretion due to the fitness cost associated with the expression of resistance (Sakoulas et al. 2003, Gill et al. 2005) and it is reflected in decreased toxin expression (Collins et al. 2010, Otto 2010). Therefore, it is not surprising that the results of the present study confirmed this by showing that virulence factor gene carriage was more diverse and abundant in MSSA than in MRSA strains. However, due to the fact that most studies on virulence factor carriage are conducted on resistant strains, there is a need for informa- tion in susceptible strains that allows a more thorough comparison. In addition, Collins et al. (2010) indicated that the fitness cost associated with resistance varies depending on the SCCmec type and antibiotic resistance levels. These authors found that in MRSA strains carrying the larger cassette SCCmec II, there was a reduction in virulence factor secretion, while strains carrying the smaller size SCCmec IV secreted a more diverse range of factors. The findings of the present study are consistent with those of Collins et al. (2010) because isolates harbouring $\mathrm{SCCmec}$ type I presented no or up to two virulence genes, whereas SCCmec type IV strains

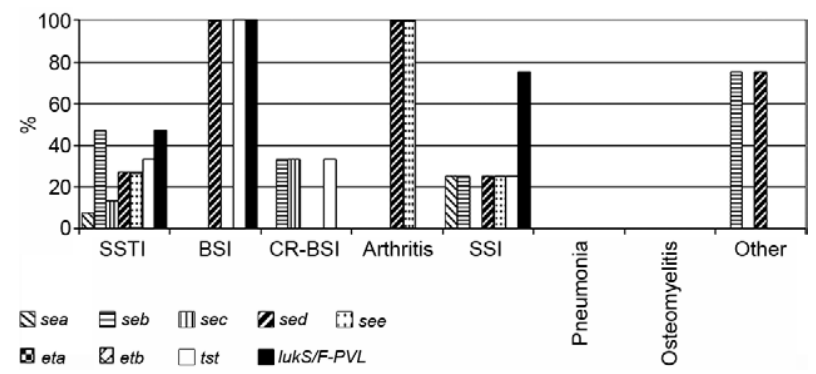

Fig. 2: percentage distribution of virulence genes encoding staphylococcal enterotoxins genes A-E (sea, seb, sec, sed and see), exfoliative toxins A and B (eta and $e t b$ ), toxic shock syndrome toxin 1 (tst) and Panton-Valentine leucocidine (lukS/F-PVL) in methicillinsusceptible Staphylococcus aureus (MSSA) according to infection types. BSI: blood stream infections; CR-BSI: catheter-related blood stream infections; other: includes urinary tract infections, conjunctivitis, meningitis and otitis; SSI: surgical site infection; SSTI: skin and soft tissue infections. 


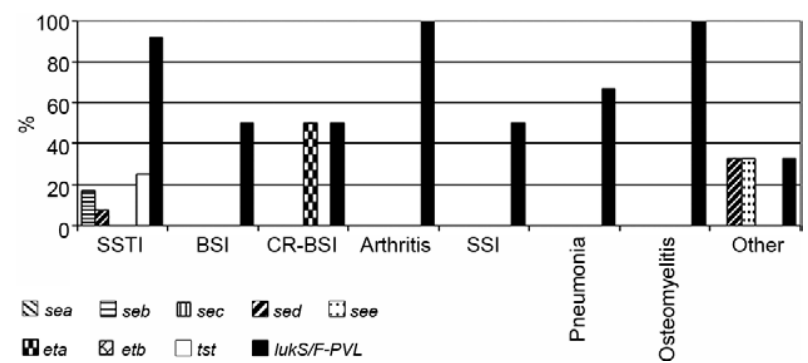

Fig. 3: percentage distribution of virulence genes encoding staphylococcal enterotoxins genes A-E (sea, seb, sec, sed and see), exfoliative toxins A and B (eta and etb), toxic shock syndrome toxin 1 ( $t s t$ ) and Panton-Valentine leukocidin (lukS/F-PV) in methicillin-resistant Staphylococcus aureus (MRSA) according to infection types. BSI: blood stream infections; CR-BSI: catheter-related blood stream infections; other: includes tracheitis, orbital cellulitis and infected pulmonary sequestration; SSI: surgical site infection; SSTI: skin and soft tissue infections.

carried one or more virulence genes simultaneously, as observed in susceptible strains. The fitness cost on the growth and cell yield of $S$. aureus containing the SCCmec elements has also been experimentally demonstrated; the higher energy demand and decreased cell yield observed in SCCmec type I strains has not been observed in the SCCmec type IV strains (Lee et al. 2007, Collins et al. 2010).

In the paediatric population evaluated, the $l u k S / F-P V$ genes were detected more commonly in MRSA isolates, whereas the enterotoxin genes were more frequently observed in MSSA strains. These results agree with those of studies that found lower frequencies of the genes encoding PVL in MSSA clinical isolates (Kuehnert et al. 2006), but contrast with data reported from the Czech Republic, where it was found that the $l u k S / F-P V$, tst and sec genes were more frequent in MSSA isolates and enterotoxins genes $s e a, s e b$, sed and eta were most prevalent in MRSA strains (Sila et al. 2009). Also, in this study, $l u k S / F-P V$ were more frequently observed in SCCmec IVc isolates (94\%), but they were not restricted to this cassette type and they were also present in SCCmec types I, IVa and V. Other studies conducted in China (Wu et al. 2010) and in USA (Abdel-Haq et al. 2009) that have evaluated the epidemiological and molecular features of MRSA and MSSA isolates from children found that the most frequent SCCmec type was IVa and all of these strains carried the $l u k S / F-P V$ genes. Also, similar to our findings, results of a study conducted in Lebanon revealed that the PVL genes were present mainly in SCCmec IVc isolates (Tokajian et al. 2010).

The low frequency of the eta and $e t b$ genes coincides with results of studies that found that $5 \%$ of the isolates harboured one or both toxins and of these, eta and etb were present in $88 \%$ and $4 \%$ of them, respectively (Ladhani et al. 1999). The low frequency of the eta and etb genes in clinical MSSA isolates was also documented in a more recent study (van Trijp et al. 2010) in which $l u k S / F-P V$ were detected in the skin and soft tissue and in other infections, such as arthritis, SSI, pneumonia and osteomyelitis. In the latter two, only the $l u k S / F-P V$ genes were detected. Although there is controversy about the role of PVL in the spread and severity of $S$. aureus infections (Oliveira et al. 2002, Chambers \& Deleo 2009, DeLeo et al. 2009, Otto 2010), the results of this study show the importance of this virulence factor among resistant strains and in different infections.

In Latin-American countries, specially Argentina and Brazil, other authors have reported the presence of PVLproducer and non-producer MRSA strains carrying SCCmec IV, which cause both community and health-care associated infections (mainly severe SSTI, bacteraemia, osteomyelitis and pneumonia or chronic lung infection) and colonise healthy adults and children (Trindade et al. 2005, de Miranda et al. 2007, Rozenbaum et al. 2009, Scribel et al. 2009, Gardella et al. 2011, Mimica et al. 2011). Nevertheless, there is little information available about the virulence factors present in the isolates and most studies do not include SCCmec typing or any molecular characterisation of the strains. In Colombia, a study conducted in Bogotá reported three paediatric cases of infections caused by PVL-positive MRSA strains carrying SCCmec IVc belonging to clonal complex 8 (CC8) with $75 \%$ similarity to USA 300 (Alvarez-Olmos et al. 2009). Remarkably, USA 300 is one of the most prevalent CAMRSA strains in the USA and it has disseminated into Canada and Europe and is implicated in SSTI with invasive disease, including severe septicaemia, necrotising pneumonia and necrotising fasciitis (Diep et al. 2006). The above suggests the possibility that the PVL-positive SCCmec IVc MRSA isolates detected in this study will be related to this $\mathrm{CC} 8$.

The findings of this study also show that MSSA strains remain an important source of infection, suggesting that MRSA has not replaced MSSA strains. Therefore, susceptible strains should continue to be monitored and controlled, as resistant strains are, due to their high pathogenic potential. In general, we observed that distinct staphylococcal toxin gene profiles were present according to methicillin resistance/susceptibility. However, future analyses of these strains should include identification of their lineage, which is necessary for better comprehension of their dynamics. This may help to determine to what extent the pathogenicity of $S$. aureus infections is influenced by the presence of staphylococcal toxins.

This is the first study conducted in a child population at the local level that shows the differences in virulence gene profiles between MRSA-MSSA hospital isolates. This information is useful for establishing effective control measures and management of infections caused by this organism. Furthermore, it is important that more comprehensive studies be conducted to evaluate the participation of microbiologic factors, such as virulence genes, in the pathogenicity of MRSA and MSSA in the community. The present study showed specific differences in the distribution of virulence genes in the $S$. aureus strains obtained from the paediatric population of a hospital in Medellín from those of other countries. This suggests that these features may vary according to geographic location and clinical-epidemiological factors, underscoring the importance of characterising the relationships between toxin genes and methicillin resistance in each region. 


\section{REFERENCES}

Abdel-Haq N, Al-Tatari H, Chearskul P, Salimnia H, Asmar BI, Fairfax MR, Amjad M 2009. Methicillin-resistant Staphylococcus aureus (MRSA) in hospitalized children: correlation of molecular analysis with clinical presentation and antibiotic susceptibility testing (ABST) results. Eur J Clin Microbiol Infect Dis 28: 547-551.

Alvarez-Olmos MI, Enriquez SP, Perez-Roth E, Mendez-Alvarez S, Escobar J, Vanegas N, Moreno J 2009. Pediatric cases from Colombia caused by a Panton-Valentine leukocidin-positive community-acquired methicillin-resistant Staphylococcus aureus ST8-SCCmec IVc clone. Pediatr Infect Dis J 28: 935.

Baker CJ 2010. Why has MRSA become such a successful pathogen? A pediatric perspective. Clinical updates in infectious diseases 13: 5231-5234.

Bocchini CE, Hulten KG, Mason EO Jr, Gonzalez BE, Hammerman WA, Kaplan SL 2006. Panton-Valentine leukocidin genes are associated with enhanced inflammatory response and local disease in acute hematogenous Staphylococcus aureus osteomyelitis in children. Pediatrics 117: 433-440.

Chambers HF 2005. Community-associated MRSA: resistance and virulence converge. $N$ Engl J Med 352: 1485-1487.

Chambers HF, Deleo FR 2009. Waves of resistance: Staphylococcus aureus in the antibiotic era. Nat Rev Microbiol 7: 629-641.

CLSI - Clinical and Laboratory Standards Institute 2010. Performance standards for antimicrobial susceptibility testing: twentieth informational supplement. Available from: clsi.org/source/ orders/free/m100-s20.pdf.

Collins J, Rudkin J, Recker M, Pozzi C, O’Gara JP, Massey RC 2010. Offsetting virulence and antibiotic resistance costs by MRSA. ISME J 4: 577-584.

de Miranda OP, Silva-Carvalho MC, Ribeiro A, Portela F, Cordeiro RP, Caetano N, Vidal CF, Figueiredo AM 2007. Emergence in Brazil of methicillin-resistant Staphylococcus aureus isolates carrying SCCmec IV that are related genetically to the USA 800 clone. Clin Microbiol Infect 13: 1165-1172.

DeLeo FR, Diep BA, Otto M 2009. Host defense and pathogenesis in Staphylococcus aureus infections. Infect Dis Clin North Am 23: 17-34.

Diep BA, Gill SR, Chang RF, Phan TH, Chen JH, Davidson MG, Lin F, Lin J, Carleton HA, Mongodin EF, Sensabaugh GF, PerdreauRemington F 2006. Complete genome sequence of USA 300, an epidemic clone of community-acquired methicillin-resistant Staphylococcus aureus. Lancet 367: 731-739.

Faden H, Rose R, Lesse A, Hollands C, Dryja D, Glick PL 2007. Clinical and molecular characteristics of staphylococcal skin abscesses in children. J Pediatr 151: 700-703.

Gardella N, Murzicato S, Di Gregorio S, Cuirolo A, Desse J, Crudo F, Gutkind G, Mollerach M 2011. Prevalence and characterization of methicillin-resistant Staphylococcus aureus among healthy children in a city of Argentina. Infect Genet Evol 11: 1066-1071.

Gill SR, Fouts DE, Archer GL, Mongodin EF, Deboy RT, Ravel J, Paulsen IT, Kolonay JF, Brinkac L, Beanan M, Dodson RJ, Daugherty SC, Madupu R, Angiuoli SV, Durkin AS, Haft DH, Vamathevan J, Khouri H, Utterback T, Lee C, Dimitrov G, Jiang L, Qin H, Weidman J, Tran K, Kang K, Hance IR, Nelson KE, Fraser CM 2005. Insights on evolution of virulence and resistance from the complete genome analysis of an early methicillinresistant Staphylococcus aureus strain and a biofilm-producing methicillin-resistant Staphylococcus epidermidis strain. J Bacteriol 187: 2426-2438.
Gordon RJ, Lowy FD 2008. Pathogenesis of methicillin-resistant Staphylococcus aureus infection. Clin Infect Dis 46 (Suppl. 5): S350-359.

Gould IM 2006. Costs of hospital-acquired methicillin-resistant Staphylococcus aureus (MRSA) and its control. Int J Antimicrob Agents 28: 379-384.

Herold BC, Immergluck LC, Maranan MC, Lauderdale DS, Gaskin RE, Boyle-Vavra S, Leitch CD, Daum RS 1998. Communityacquired methicillin-resistant Staphylococcus aureus in children with no identified predisposing risk. JAMA 279: 593-598.

John CC, Schreiber JR 2006. Therapies and vaccines for emerging bacterial infections: learning from methicillin-resistant Staphylococcus aureus. Pediatr Clin North Am 53: 699-713.

Kim JS, Song W, Kim HS, Cho HC, Lee KM, Choi MS, Kim EC 2006. Association between the methicillin resistance of clinical isolates of Staphylococcus aureus, their staphylococcal cassette chromosome mec ( $\mathrm{SCCmec}$ ) subtype classification and their toxin gene profiles. Diagn Microbiol Infect Dis 56: 289-295.

Kondo Y, Ito T, Ma XX, Watanabe S, Kreiswirth BN, Etienne J, Hiramatsu K 2007. Combination of multiplex PCRs for staphylococcal cassette chromosome mec type assignment: rapid identification system for $m e c, c c r$ and major differences in junkyard regions. Antimicrob Agents Chemother 51: 264-274.

Kuehnert MJ, Kruszon-Moran D, Hill HA, McQuillan G, McAllister SK, Fosheim G, McDougal LK, Chaitram J, Jensen B, Fridkin SK, Killgore G, Tenover FC 2006. Prevalence of Staphylococcus aureus nasal colonization in the United States, 2001-2002. J Infect Dis 193: 172-179.

Ladhani S, Joannou CL, Lochrie DP, Evans RW, Poston SM 1999. Clinical, microbial and biochemical aspects of the exfoliative toxins causing staphylococcal scalded-skin syndrome. Clin Microbiol Rev 12: 224-242.

Lee GM, Huang SS, Rifas-Shiman SL, Hinrichsen VL, Pelton SI, Kleinman K, Hanage WP, Lipsitch M, McAdam AJ, Finkelstein JA 2009. Epidemiology and risk factors for Staphylococcus aureus colonization in children in the post-PCV7 era. BMC Infect Dis 9: 110.

Lee SM, Ender M, Adhikari R, Smith JM, Berger-Bachi B, Cook GM 2007. Fitness cost of staphylococcal cassette chromosome mec in methicillin-resistant Staphylococcus aureus by way of continuous culture. Antimicrob Agents Chemother 51: 1497-1499.

McClure JA, Conly JM, Lau V, Elsayed S, Louie T, Hutchins W, Zhang K 2006. Novel multiplex PCR assay for detection of the staphylococcal virulence marker Panton-Valentine leukocidin genes and simultaneous discrimination of methicillin-susceptible from -resistant staphylococci. J Clin Microbiol 44: 1141-1144.

Mehrotra M, Wang G, Johnson WM 2000. Multiplex PCR for detection of genes for Staphylococcus aureus enterotoxins, exfoliative toxins, toxic shock syndrome toxin 1 , and methicillin resistance. J Clin Microbiol 38: 1032-1035.

Miller LG, Diep BA 2008. Clinical practice: colonization, fomites and virulence: rethinking the pathogenesis of community-associated methicillin-resistant Staphylococcus aureus infection. Clin Infect Dis 46: 752-760.

Mimica MJ, Berezin EN, Damaceno N, Carvalho RB 2011. SCCmec type IV, PVL-negative, methicillin-resistant Staphylococcus aureus in cystic fibrosis patients from Brazil. Curr Microbiol 62: 388-390.

Oliveira DC, Tomasz A, de Lencastre H 2002. Secrets of success of a human pathogen: molecular evolution of pandemic clones of methicillin-resistant Staphylococcus aureus. Lancet Infect Dis 2: 180-189.

Otto M 2010. Basis of virulence in community-associated methicillinresistant Staphylococcus aureus. Annu Rev Microbiol 64: 143-162.

Rozenbaum R, Sampaio MG, Batista GS, Garibaldi AM, Terra GM, Souza MJ, Vieira EN, Silva-Carvalho MC, Teixeira LA, Figueire- 
do AM 2009. The first report in Brazil of severe infection caused by community-acquired methicillin-resistant Staphylococcus aureus (CA-MRSA). Braz J Med Biol Res 42: 756-760.

Sakoulas G, Eliopoulos GM, Moellering RC Jr, Novick RP, Venkataraman L, Wennersten C, DeGirolami PC, Schwaber MJ, Gold HS 2003. Staphylococcus aureus accessory gene regulator (agr) group II: is there a relationship to the development of intermediate-level glycopeptide resistance? J Infect Dis 187: 929-938.

Scribel LV, Silva-Carvalho MC, Souza RR, Superti SV, Kvitko CH, Figueiredo AM, Zavascki AP 2009. Clinical and molecular epidemiology of methicillin-resistant Staphylococcus aureus carrying SCCmec IV in a university hospital in Porto Alegre, Brazil. Diagn Microbiol Infect Dis 65: 457-461.

Sila J, Sauer P, Kolar M 2009. Comparison of the prevalence of genes coding for enterotoxins, exfoliatins, panton-valentine leukocidin and $t s t-1$ between methicillin-resistant and methicillin-susceptible isolates of Staphylococcus aureus at the university hospital in Olomouc. Biomed Pap Med Fac Univ Palacky Olomouc Czech Repub 153: 215-218.

Stankovic C, Mahajan PV 2006. Healthy children with invasive community-acquired methicillin-resistant Staphylococcus aureus infections. Pediatr Emerg Care 22: 361-363.
Tokajian ST, Khalil PA, Jabbour D, Rizk M, Farah MJ, Hashwa FA, Araj GF 2010. Molecular characterization of Staphylococcus aureus in Lebanon. Epidemiol Infect 138: 707-712.

Trindade PA, Pacheco RL, Costa SF, Rossi F, Barone AA, Mamizuka EM, Levin AS 2005. Prevalence of SCCmec type IV in nosocomial bloodstream isolates of methicillin-resistant Staphylococcus aureus. J Clin Microbiol 43: 3435-3437.

Tristan A, Ferry T, Durand G, Dauwalder O, Bes M, Lina G, Vandenesch F, Etienne J 2007. Virulence determinants in community and hospital methicillin-resistant Staphylococcus aureus. J Hosp Infect 65 (Suppl. 2): 105-109.

van Trijp MJ, Melles DC, Snijders SV, Wertheim HF, Verbrugh HA, van Belkum A, van Wamel WJ 2010. Genotypes, superantigen gene profiles and presence of exfoliative toxin genes in clinical methicillin-susceptible Staphylococcus aureus isolates. Diagn Microbiol Infect Dis 66: 222-224.

Wu D, Wang Q, Yang Y, Geng W, Yu S, Yao K, Yuan L, Shen X 2010. Epidemiology and molecular characteristics of community-associated methicillin-resistant and methicillin-susceptible Staphylococcus aureus from skin/soft tissue infections in a children's hospital in Beijing, China. Diagn Microbiol Infect Dis 67: 1-8. 